Artículo

\title{
Nutrición mineral del chilhuacle en tres etapas fenológicas
}

\author{
Evelia Fajardo-Rebollar ${ }^{1}$ \\ Oscar Gabriel Villegas-Torres ${ }^{1 \S}$ \\ María Andrade-Rodríguez ${ }^{1}$ \\ Héctor Sotelo-Nava ${ }^{1}$ \\ Francisco Perdomo Roldán ${ }^{1,2}$ \\ José Luis Viveros-Ceballos ${ }^{1,3}$
}

${ }^{1}$ Facultad de Ciencias Agropecuarias-Universidad Autónoma del Estado de Morelos. ${ }^{2}$ Centro de Estudios Superiores de Xalostoc. ${ }^{3}$ Centro de Investigaciones Químicas. Av. Universidad núm. 1001, Chamilpa, Cuernavaca, Morelos, México. CP. 62209. (fajardorebollar@gmail.com; maria.andrade@uaem.mx; hector.sotelo@uaem.mx; fperdomor@gmail.com; jlvc@uaem.mx).

§Autor para correspondencia: voscar66@yahoo.com.mx.

\section{Resumen}

El chilhuacle (Capsicum annuum L.) es uno de los chiles más populares en Oaxaca. Sin embargo, se desconocer el manejo y la poscosecha de los frutos afecta directamente el rendimiento y la calidad del fruto. Con la finalidad de evaluar diferentes soluciones nutritivas para cada etapa fenológica, se establecieron tres experimentos en el campo experimental de la Facultad de Ciencias Agropecuarias de la Universidad Autónoma del Estado de Morelos. En la etapa vegetativa se evaluaron tres concentraciones de $\mathrm{NO}_{3}{ }^{-}$, en la etapa reproductiva nueve relaciones $\mathrm{NO}_{3}{ }^{-}: \mathrm{H}_{2} \mathrm{PO}_{4}^{-}: \mathrm{SO}_{4}{ }^{2-}$, y en la etapa de fructificación nueve relaciones $\mathrm{NO}_{3}^{-}: \mathrm{K}^{+}$. Los tratamientos fueron distribuidos en un diseño de bloques completamente al azar con cinco repeticiones y una planta por unidad experimental en contenedores de polietileno negro (15.14 L), un sistema de siembra tresbolillo (50 cm etapa vegetativa y $75 \mathrm{~cm}$ en la etapa reproductiva y fructificación), sistema de riego por goteo y grava de tezontle rojo como sustrato. Los resultados indicaron que las soluciones nutritivas produjeron significancia en la altura, diámetro de tallo, volumen de raíz, materia seca de tallo y planta completa 14 meq $\mathrm{L}^{-1}{\mathrm{de} \mathrm{NO}_{3}}^{-}$ en la etapa vegetativa; redujo el aborto floral e incrementó el número de botones por planta con 10:0.75:9.25 meq $\mathrm{L}^{-1}$ de $\mathrm{NO}_{3}{ }^{-}: \mathrm{H}_{2} \mathrm{PO}_{4}{ }^{-}: \mathrm{SO}_{4}{ }^{2-}$ durante la etapa reproductiva y en fruto, la relación 14:9 meq $\mathrm{L}^{-1}$ de $\mathrm{NO}_{3}^{-}: \mathrm{K}^{+}$favoreció al incrementar significativamente el diámetro ecuatorial, peso de materia fresca y seca de fruto.

Palabras clave: etapa de fructificación, fenología, soluciones nutritivas.

Recibido: diciembre de 2021

Aceptado: febrero de 2022 


\section{Introducción}

El chilhuacle (Capsicum annuum L.) negro es un fruto endémico de San Juan Bautista Cuicatlán, Oaxaca (López et al., 2016), es de forma trapezoidal de $10 \mathrm{~cm}$ de largo y $8 \mathrm{~cm}$ de ancho, en estado inmaduro es color verde intenso, en estado maduro es café brillante y negro al deshidratarse (López y Pérez, 2015), se comercializa principalmente en seco empleado como ingrediente principal para elaborar el 'mole negro oaxaqueño' (García et al., 2017), uno de los siete moles más populares del país donde se encuentra citado en los libros más antiguos de gastronomía, además mantiene reconocimiento nacional e internacional al servirse como un platillo gourmet.

En los últimos años, el precio del fruto seco se ha elevado debido a los escases de productores de chilhuacle, incremento de los costos de producción, particularmente por el uso excesivo de agroquímicos para el control de plagas y enfermedades (López et al., 2016) y el desconocimiento de la nutrición del cultivo, lo cual es de gran relevancia porque una planta bien nutrida es más tolerante al ataque de plagas, enfermedades y virus (Sieiro et al., 2020) que en conjunto es consecuencia de un bajo rendimiento. La fertilización solo sucede en dos o tres ocasiones durante el ciclo, donde la fertilización foliar es complementaria y más concurrida, si las condiciones lo permiten se alcanza una cosecha estimada de $1 \mathrm{t} \mathrm{ha}^{-1}$ (López et al., 2016).

La formulación de soluciones nutritivas en cada etapa fenológica se realiza con la finalidad de aportar los nutrimentos necesarios para incrementar el crecimiento vegetal, rendimiento, calidad de los órganos de interés y en el caso de las hortalizas de fruto reducir el aborto floral (MorenoPérez et al., 2019). Las soluciones nutritivas no solo inducen a lograr el máximo potencial productivo en un cultivo (Luna-Fletes et al., 2021) o disminuir los daños que provocan la aparición de patógenos que afectan directamente la calidad comercial de los frutos (Fajardo et al., 2021), sino repercute en el desarrollo, rendimiento y calidad de los frutos (López-Gómez et al., 2017).

De los macronutrientes indispensables en los cultivos, el nitrógeno es el nutriente con mayor demanda por las plantas, se relaciona con el crecimiento, desarrollo (Larios-González et al., 2021) y rendimiento en los cultivos. En la etapa de formación de flores y amarre de frutos, aparte de la necesidad de $\mathrm{N}$, el $\mathrm{P}$ participa en la formación de raíces, flores, frutos y maduración de semillas (Taiz y Zeiger, 2010), mientras que el $\mathrm{S}$ en forma de $\mathrm{SO}_{4}{ }^{2-}$ contribuye en la diferenciación de yemas florales (Corrales-Maldonado et al., 2014), estimula el crecimiento, incrementa la calidad y uniformidad de los frutos, favoreciendo la resistencia al frío, tolerancia a sequía y sales (Nazar et al., 2011). En la etapa de fructificación, el potasio (K) está relacionado con la calidad y el rendimiento de los cultivos (Pavón et al., 2021), incrementando la vitamina C, el color y sabor de las frutas, mayor tamaño y vida de anaquel.

Debido a los requerimientos nutrimentales específicos durante el desarrollo de las especies y variedades vegetales, el objetivo de esta investigación fue evaluar el efecto de soluciones nutritivas de diferente composición química en tres etapas fenológicas del chilhuacle (vegetativa, reproductiva y fructificación) con la finalidad de favorecer el crecimiento, rendimiento y calidad de los frutos. 


\section{Materiales y métodos}

\section{Ubicación de los experimentos}

Se realizaron tres experimentos, uno por cada etapa fenológica del chilhuacle (vegetativa, reproductiva y fructificación), en un invernadero de cubierta de polietileno blanco $30 \%$ sombra en el campo experimental de la Facultad de Ciencias Agropecuarias de la Universidad Autónoma del Estado de Morelos, Cuernavaca, Morelos.

\section{Material vegetal}

Se utilizaron semillas criollas de chilhuacle negro provenientes de productores de Oaxaca, mismas que se sembraron en charolas de polietileno de 200 cavidades en un sustrato comercial formulado para almácigo a base de turba (Sunshine $3^{\circledR}$ ), el suministro de nutrimentos se realizó con la solución nutritiva universal (Steiner, 1984). Para la obtención de semilla, se seleccionan frutos del ciclo anterior de apariencia sana (libre de enfermedades), uniformes y con tamaño promedio, normalmente de $6 \mathrm{~cm}$ de largo, $5 \mathrm{~cm}$ de ancho. El peso de los frutos en estado fresco es de $40 \mathrm{~g} \mathrm{y}$ $5.6 \mathrm{~g}$ en completa deshidratación. El rendimiento por planta de $1.4 \mathrm{~kg}$ en fruto fresco y en seco oscila entre los $250 \mathrm{~g} \mathrm{planta}^{-1}$.

\section{Unidad experimental}

Cada experimento se realizó en sistema hidropónico de circuito abierto con riego por goteo. Se utilizaron contenedores de polietileno negro $(15.14 \mathrm{~L})$ y tezontle rojo con granulometría menor a $0.5 \mathrm{~mm}$ de diámetro como sustrato. En los tres casos, se tuvo una planta de chilhuacle negro por contenedor. La distancia entre plantas varió en función de la etapa fenológica $(50 \mathrm{~cm}$ para la vegetativa y $70 \mathrm{~cm}$ para la reproductiva y fructificación) en arreglo topológico tres bolillos, manteniendo tres módulos para cada experimento. La unidad experimental en la etapa vegetativa se conformó de seis plantas (una planta por contenedor), la unidad de muestreo fueron las dos plantas centrales, se descartaron las plantas laterales para reducir la interacción entre las mismas. Para la etapa reproductiva y fructificación los módulos fueron diseñados con una distancia de 70 $\mathrm{cm}$ entre planta y planta donde la unidad de muestreo fue una planta por contenedor siendo cinco repeticiones por tratamiento.

\section{Manejo de los experimentos}

La fase de almácigo, correspondiente a la etapa vegetativa, tuvo su propio manejo agronómico y no fue motivo de evaluación. El tiempo de trasplante fue determinado por el número de hojas desarrolladas. A partir de la décima hoja (73 dds), las plántulas se encuentran dentro de las condiciones óptimas para poder ser trasplantadas. De esta manera, el primer experimento (etapa vegetativa) inició en el día 74 dds (cuando 50\% de las plántulas presentaron la décima hoja verdadera con una longitud de $5 \pm 1 \mathrm{~mm}$ ) y finalizó a los 29 ddt cuando $50 \%$ de las plantas presentaron el primer botón floral $(5 \pm 1 \mathrm{~mm})$.

El segundo experimento (etapa reproductiva), inició cuando el 50\% de las plantas presentaron visualmente el primer botón floral $(5 \pm 1 \mathrm{~mm})$ y finalizó a los 40 ddt (11 días) en el momento que el $50 \%$ de las plantas tuvieron el primer fruto $(10 \pm 1 \mathrm{~mm})$ en este caso, la nutrición de la etapa 
vegetativa fue con la solución nutritiva universal (SNU) (Steiner, 1984). El tercer experimento correspondiente a la etapa de fructificación inició a los 41 ddt cuando $50 \%$ de las plantas presentaron el primer fruto y finalizó a los 184 ddt hasta la evaluación de último fruto cosechado, en las etapas vegetativa y floración se aplicó la SNU. Los frutos se cosecharon durante 18 semanas, cuando $100 \%$ del exocarpo presentó madurez comercial, de acuerdo con García-Gaytán et al. (2017) la maduración de fruto sucede entre los 60 hasta 78 días después de la antesis.

\section{Tratamientos y diseño experimental}

El diseño de las soluciones nutritivas evaluadas en cada etapa fenológica se realizó considerando la solución nutritiva universal (Steiner, 1984) como control y referencia para el diseño de las soluciones nutritivas. Los testigos fueron: 12 meq $\mathrm{L}^{-1}$ de $\mathrm{NO}_{3}{ }^{-}$en la etapa vegetativa, 12:1:7 meq $\mathrm{L}^{-1}$ de $\mathrm{NO}_{3}^{-}: \mathrm{H}_{2} \mathrm{PO}_{4}^{-}: \mathrm{SO}_{4}{ }^{2-}$ en la etapa reproductiva y $12: 7$ meq $\mathrm{L}^{-1} \mathrm{de} \mathrm{NO}_{3}^{-}: \mathrm{K}^{+}$en la de fructificación. La solución nutritiva universal de Steiner mantiene las relaciones mutuas entre aniones y cationes, la concentración iónica total y el pH, como factores importantes que darán respuesta positiva en el crecimiento y desarrollo de las plantas, considerada como referencia para una nutrición general de cualquier cultivo.

En la etapa vegetativa se tuvieron tres soluciones nutritivas resultado de modificar la concentración de $\mathrm{NO}_{3}{ }^{-}\left(10,12\right.$ y 14 meq $\left.\mathrm{L}^{-1}\right)$, en la reproductiva se generaron nueve soluciones nutritivas con diferentes relaciones $\mathrm{NO}_{3}^{-}: \mathrm{H}_{2} \mathrm{PO}_{4}{ }^{-}: \mathrm{SO}_{4}{ }^{2-}$, las cuales fueron el resultado de combinar 10, 12 y 14 meq $\mathrm{L}^{-1}$ de $\mathrm{NO}_{3}{ }^{-}$con $0.75,1$ y 1.25 meq $\mathrm{L}^{-1} \mathrm{de}_{2} \mathrm{PO}_{4}^{-}$, mientras que en la de fructificación se evaluaron nueve soluciones nutritivas como consecuencia de la combinación de 10, 12 y 14 meq $\mathrm{L}^{-1}$ de $\mathrm{NO}_{3}{ }^{-}$con 5,7 y 9 meq L ${ }^{-1}$ de $\mathrm{K}^{+}$. En los tres experimentos, el diseño experimental fue bloques completos al azar, con cinco repeticiones por tratamiento.

\section{Variables de respuesta}

En los tres experimentos (vegetativa, reproductiva y fructificación) se evaluaron las variables ambientales como temperatura, humedad relativa e intensidad luminosa desde el trasplante hasta la evaluación medidas con un $\mathrm{Hobo}^{\circledR}$ data logger.

\section{Etapa vegetativa}

Las variables de respuesta se evaluaron cuando $50 \%$ de las plantas presentaron el primer botón floral $(5 \pm 1 \mathrm{~mm})$. Se determinaron: altura de la planta con un flexómetro (Pretul ${ }^{\circledR}$ ) desde la base del tallo principal hasta el ápice del tallo más largo, diámetro del tallo principal se realizó un corte a una distancia de $2 \mathrm{~cm}$ por encima del sustrato con un vernier digital (Stainless Hardened ${ }^{\circledR}$ ) (López-Gómez et al., 2017), contenido relativo de clorofila (CRC) con SPAD 502 Plus (Minolta ${ }^{\circledR}$ ) en hojas recientemente maduras, área foliar determinada con un integrador de área foliar (LICOR, LI-3100), longitud de la raíz más larga medida con un flexómetro y volumen de raíz por el método de desplazamiento de agua, materia seca de los órganos de la planta (raíz, tallo y hojas), para ello se utilizó una estufa de circulación forzada de aire (Luzeren, DGH9070A) a una temperatura de 65 ${ }^{\circ} \mathrm{C}$ durante $72 \mathrm{~h}$ y una balanza digital. 


\section{Etapa reproductiva}

Las variables de respuesta se evaluaron cuando $50 \%$ de las plantas presentaron el primer fruto con una longitud de $10 \pm 1 \mathrm{~mm}$. La altura de la planta, diámetro del tallo principal, contenido relativo de clorofila, área foliar, volumen de raíz, peso de materia seca de raíz, tallo y hojas se determinaron de la misma manera que en la etapa vegetativa. En este caso también se consideraron botones por planta, flores por planta y porcentaje de aborto floral.

\section{Etapa de fructificación}

Las variables de crecimiento de las pantas se evaluaron al finalizar la cosecha, de igual forma que en la etapa vegetativa (área foliar estimada, contenido relativo de clorofila, peso de materia seca de raíz, tallo y hojas). El área foliar y la materia seca de las hojas fue estimada considerando los entrenudos del 10 al 15 de los dos tallos principales y secundarios. También se determinó la longitud y diámetro del fruto medido con vernier digital (Stainless Hardened ${ }^{\circledR}$ ) con base en la Norma NNX-FF-107/1-SCFI-2006, grosor del exocarpio (promedio de dos puntos opuestos en el centro del fruto) con vernier digital (Stainless Hardened ${ }^{\circledR}$ ), sólidos solubles totales $\left({ }^{\circ}\right.$ Brix) cuantificados con un refractómetro portátil (Atago ${ }^{\circledR}$, PAL-1 3810), materia fresca y seca de cada fruto (etapa vegetativa), semillas por fruto, número de frutos por planta, frutos de los tallos principales, porcentaje de aborto floral, rendimiento de materia fresca y seca de frutos por planta.

\section{Análisis estadístico}

Los datos obtenidos se sometieron al análisis de varianza con el programa SAS (versión 9.0) y a los que presentaron diferencia estadística se les realizó la prueba de comparación múltiple de medias LSD $(p \leq 0.05)$.

\section{Resultados y discusión}

La etapa vegetativa duró 29 días, con temperatura promedio de $21.4{ }^{\circ} \mathrm{C}$ y humedad relativa de 49.5\%, la cual puede considerarse de corta duración ya que Azofeifa y Moreira (2004) mencionan 40 días para el chile jalapeño y Noh et al. (2010) indican 50 días en chile habanero. Dentro de los C. annuиm, las variedades genéticamente no manipuladas su crecimiento y comportamiento puede ser variable, por lo que en chilhuacle el tiempo de trasplante puede no ser constante por efecto de las condiciones climáticas que se cultive. En programas de nutrición es importante considerar la duración de la etapa vegetativa de acuerdo con la especie y variedad ya que algunas características importantes son la división celular y rápido crecimiento de raíces, tallos y hojas, implicando distribución de biomasa a estos órganos y demanda de nutrimentos (Azofeifa y Moreira, 2004), como el $\mathrm{N}$ al ser el elemento responsable del crecimiento y desarrollo (Larios-González et al., 2021) sobre todo en los primeros días después del trasplante donde el crecimiento es acelerado.

Con relación al efecto de la concentración de $\mathrm{NO}_{3}{ }^{-}$en la solución nutritiva en el crecimiento de las plantas de chilhuacle, 10,12 y $14 \mathrm{meq} \mathrm{L}^{-1}$ tuvieron efecto estadísticamente similar $(p \leq 0.05)$ en la concentración relativa de clorofila (50.35 unidades SPAD), área foliar $\left(292.4 \mathrm{~cm}^{2}\right)$ y longitud de raíz $(18 \mathrm{~cm})$, al igual que en la acumulación de materia seca de raíz $(0.5 \mathrm{~g})$ y hoja $(1.23 \mathrm{~g})$. En las variables altura de planta, diámetro de tallo principal, volumen de raíz, materia seca de tallo y de 
la planta completa si resultaron afectadas por la concentración de $\mathrm{NO}_{3}{ }^{-}$de la solución nutritiva (Cuadro 1). En este sentido, con 14 meq $\mathrm{L}^{-1}$ de $\mathrm{NO}_{3}{ }^{-}$las plantas fueron $11.51 \%$ más altas, se observó un incremento de $21 \%$ más en el diámetro de tallo a diferencia de las plantas nutridas con 12 meq $\mathrm{L}^{-1}$ (testigo), algo similar en el volumen de raíz con $14 \mathrm{meq} \mathrm{L}^{-1}{\mathrm{de} \mathrm{NO}_{3}}^{-}$las plantas presentaron

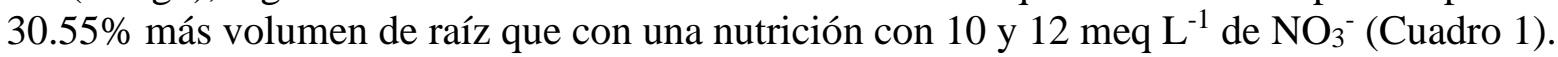

Con relación a la acumulación de materia seca, en tallo se incrementó $40.5 \%$ con 14 meq $\mathrm{L}^{-1}$ en comparación con las plantas nutridas con 12 meq $\mathrm{L}^{-1}$ de $\mathrm{NO}_{3}{ }^{-}$. Al considerar la materia seca total, las plantas nutridas con 14 meq $\mathrm{L}^{-1} \mathrm{de} \mathrm{NO}_{3}{ }^{-}$produjeron $22.63 \%$ a diferencia de una nutrición con 12 meq L $\mathrm{L}^{-1}$; sin embargo, con 10 meq $\mathrm{L}^{-1}$ la producción de materia seca aumentó en $10.28 \%$ que el tratamiento control (Cuadro 1).

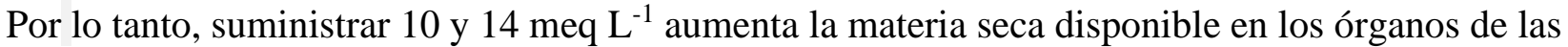
plantas de chilhuacle, donde dicho parámetro nos indica la cantidad de materia acumulable para en los órganos evaluados. Las plantas sobresalientes en relación con altura, diámetro de tallo, volumen de raíz, materia seca de tallo y planta completa (Cuadro 1) destinaron $45 \%$ de su materia seca a las hojas, $37 \%$ al tallo y $18 \%$ a raíz, una distribución equilibrada de materia seca entre la parte área en mayor proporción y menos a tallos y raíces (Peil y Gálvez, 2005).

Cuadro 1. Efecto de la concentración de $\mathrm{NO}_{3}{ }^{-}$de la solución nutritiva suministrada durante la etapa vegetativa del chilhuacle sobre variables morfológicas y producción de materia seca de las plantas.

\begin{tabular}{cccccc}
\hline $\begin{array}{c}\text { Concentración de } \mathrm{NO}_{3}{ }^{-} \\
(\mathrm{meq} \mathrm{L})\end{array}$ & $\mathrm{AP}(\mathrm{cm})$ & $\mathrm{DTP}(\mathrm{mm})$ & $\mathrm{VR}\left(\mathrm{cm}^{3}\right)$ & MST $(\mathrm{g})$ & MSTOT $(\mathrm{g})$ \\
\hline 10 & $32.3 \mathrm{ab}$ & $4.57 \mathrm{ab}$ & $7 \mathrm{~b}$ & $0.96 \mathrm{ab}$ & $2.68 \mathrm{ab}$ \\
$12^{*}$ & $30.4 \mathrm{~b}$ & $4.14 \mathrm{~b}$ & $7.4 \mathrm{~b}$ & $0.79 \mathrm{~b}$ & $2.43 \mathrm{~b}$ \\
14 & $33.9 \mathrm{a}$ & $5.01 \mathrm{a}$ & $9.4 \mathrm{a}$ & $1.11 \mathrm{a}$ & $2.98 \mathrm{a}$ \\
$\mathrm{DMS}$ & 1.92 & 0.71 & 1.36 & 0.15 & 0.42 \\
$\mathrm{CV}(\%)$ & 6.43 & 16.82 & 18.51 & 17.58 & 17.01 \\
\hline
\end{tabular}

Medias con misma literal en la columna son iguales estadísticamente de acuerdo con la prueba LSD $(p \leq 0.05)$. AP= altura de planta; $\mathrm{DTP}=$ diámetro de tallo principal; $\mathrm{VR}=$ volumen de raíz; MST= materia seca de tallo; MSTOT= materia seca de la planta completa; $\mathrm{DMS}=$ diferencia mínima significativa; $\mathrm{CV}=$ coeficiente de variación; ${ }^{*}=$ tratamiento testigo $\left(12 \mathrm{meq} \mathrm{L}^{-1}\right)$.

De acuerdo con el análisis de varianza $(p \leq 0.05)$ la altura de planta, diámetro de tallo principal, contenido relativo de clorofila y área foliar, las plantas de chilhuacle nutridas con 10:0.75:9.25 y el testigo (12:1:7) fueron similares estadísticamente en cuanto al crecimiento durante el periodo que fueron cultivadas.

Las relaciones $\mathrm{NO}_{3}{ }^{-}: \mathrm{H}_{2} \mathrm{PO}_{4}{ }^{-}: \mathrm{SO}_{4}{ }^{2-}$ suministradas al chilhuacle durante la etapa de floración tuvieron efecto estadísticamente diferente en las variables de crecimiento y acumulación de materia seca (Cuadro 2). En la etapa floración, las variables de mayor importancia fueron el número de flores y botones. En promedio, las plantas de chilhuacle presentaron 3.4 flores/planta independientemente de la relación nutritiva que fueron tratadas. En cuanto al número de botones por planta, la relación $\mathrm{NO}_{3}{ }^{-}: \mathrm{H}_{2} \mathrm{PO}_{4}^{-}: \mathrm{SO}_{4}{ }^{2-}$ 10:0.75:9.25 meq L ${ }^{-1}$ y el testigo fueron estadísticamente similares $(p \leq 0.05)$ presentando la misma cantidad de botones en un $30.55 \%$ más con respecto a la relación con 14:0.75:5.25 meq $\mathrm{L}^{-1}$. 
En este mismo sentido, al aumentar el número de botones por planta no aseguramos el desarrollo de frutos, pero disminuye el aborto de flor al menos 2.05\% (Cuadro 2). Por lo tanto, el N y P a 10 y 0.75 meq $\mathrm{L}^{-1}$ y 9.25 meq $\mathrm{L}^{-1}$ de $\mathrm{S}$ reduce el porcentaje de aborto y aumenta el número de botones por planta, encontrando que la disponibilidad de $\mathrm{N}$ en la planta depende de la formación de botones y el desarrollo de flores, además el $\mathrm{SO}_{4}{ }^{2-}$ participa en la formación de yemas florales (CorralesMaldonado et al., 2014). Por lo tanto, conforme incrementa la concentración de $\mathrm{NO}_{3}{ }^{-}$, aumente el aborto de flor y disminuya el número de frutos producidos por planta.

Cuadro 2. Efecto de las relaciones $\mathrm{NO}_{3}{ }^{-}: \mathrm{H}_{2} \mathrm{PO}_{4}{ }^{-}: \mathrm{SO}_{4}{ }^{2-}$ de la solución nutritiva suministrada en la etapa reproductiva del chilhuacle sobre variables morfológicas y producción de materia seca de las plantas.

\begin{tabular}{cccccccccccc}
\hline $\begin{array}{c}\text { Relaciones } \\
\mathrm{NO}_{3}^{-}: \mathrm{H}_{2} \mathrm{PO}_{4}^{-}: \mathrm{SO}_{4}^{2-} \\
(\mathrm{meq} \mathrm{L})\end{array}$ & $\begin{array}{c}\mathrm{AP} \\
(\mathrm{cm})\end{array}$ & $\begin{array}{c}\mathrm{DTP} \\
(\mathrm{mm})\end{array}$ & $\mathrm{CRC}$ & $\begin{array}{c}\mathrm{AF} \\
\left(\mathrm{cm}^{2}\right)\end{array}$ & $\begin{array}{c}\mathrm{VR} \\
\left(\mathrm{cm}^{3}\right)\end{array}$ & $\begin{array}{c}\text { NBP } \\
\text { PAF }\end{array}$ & $\begin{array}{c}\text { MSR } \\
(\mathrm{g})\end{array}$ & $\begin{array}{c}\text { MST } \\
(\mathrm{g})\end{array}$ & $\begin{array}{c}\text { MSH } \\
(\mathrm{g})\end{array}$ & $\begin{array}{c}\text { MSTOT } \\
(\mathrm{g})\end{array}$ \\
\hline $10: 0.75: 9.25$ & $48.92 \mathrm{ab}$ & $6.39 \mathrm{a}$ & $57.04 \mathrm{ab}$ & $844.8 \mathrm{ab}$ & $10 \mathrm{c}$ & $14 \mathrm{a}$ & 2.05 & $1.51 \mathrm{ab}$ & $4.1 \mathrm{ab}$ & $3.84 \mathrm{a}$ & $9.46 \mathrm{ab}$ \\
$10: 1: 9$ & $44.66 \mathrm{~b}$ & $5.77 \mathrm{ab}$ & $56.82 \mathrm{ab}$ & $790.4 \mathrm{ab}$ & $11 \mathrm{c}$ & $12 \mathrm{ab}$ & 2.83 & $1.28 \mathrm{~b}$ & $3.52 \mathrm{~b}$ & $3.45 \mathrm{ab}$ & $8.26 \mathrm{~b}$ \\
$10: 1.25: 8.75$ & $48.12 \mathrm{ab}$ & $6.4 \mathrm{a}$ & $59.5 \mathrm{a}$ & $921.8 \mathrm{ab}$ & $15.6 \mathrm{ab}$ & $13.2 \mathrm{ab}$ & 2.64 & $1.98 \mathrm{a}$ & $4.43 \mathrm{a}$ & $3.97 \mathrm{a}$ & $10.4 \mathrm{a}$ \\
$12: 0.75: 7.25$ & $48.06 \mathrm{ab}$ & $6.03 \mathrm{ab}$ & $58.06 \mathrm{ab}$ & $761 \mathrm{ab}$ & $14 \mathrm{abc}$ & $12.6 \mathrm{ab}$ & 5.11 & $1.45 \mathrm{~b}$ & $3.76 \mathrm{ab}$ & $3.6 \mathrm{ab}$ & $8.82 \mathrm{ab}$ \\
$12: 1: 7^{*}$ & $49.72 \mathrm{a}$ & $5.96 \mathrm{ab}$ & $54.94 \mathrm{~b}$ & $959 \mathrm{a}$ & $16.8 \mathrm{a}$ & $14.2 \mathrm{a}$ & 7.03 & $1.59 \mathrm{ab}$ & $4.18 \mathrm{ab}$ & $3.99 \mathrm{a}$ & $9.77 \mathrm{ab}$ \\
$12: 1.25: 6.75$ & $48.22 \mathrm{ab}$ & $6.11 \mathrm{ab}$ & $56.46 \mathrm{ab}$ & $808.1 \mathrm{ab}$ & $11.6 \mathrm{abc}$ & $11.6 \mathrm{ab}$ & 7.17 & $1.31 \mathrm{~b}$ & $3.92 \mathrm{ab}$ & $3.55 \mathrm{ab}$ & $8.8 \mathrm{ab}$ \\
$14: 0.75: 5.25$ & $48.62 \mathrm{ab}$ & $6 \mathrm{ab}$ & $58.1 \mathrm{ab}$ & $711.8 \mathrm{~b}$ & $14.6 \mathrm{abc}$ & $10.8 \mathrm{~b}$ & 4.13 & $1.45 \mathrm{~b}$ & $4.26 \mathrm{ab} 3.65 \mathrm{ab}$ & $9.37 \mathrm{ab}$ \\
$14: 1: 5$ & $46.62 \mathrm{ab}$ & $6.24 \mathrm{ab}$ & $55.7 \mathrm{ab}$ & $718.1 \mathrm{~b}$ & $11.8 \mathrm{bc}$ & $11.8 \mathrm{ab}$ & 6.61 & $1.25 \mathrm{~b}$ & $3.63 \mathrm{ab}$ & $3.13 \mathrm{~b}$ & $8.03 \mathrm{~b}$ \\
$14: 1.25: 4.75$ & $45.08 \mathrm{ab}$ & $5.51 \mathrm{~b}$ & $58.88 \mathrm{ab}$ & $759.3 \mathrm{ab}$ & $9.6 \mathrm{c}$ & $11.8 \mathrm{ab}$ & 5.53 & $1.33 \mathrm{~b}$ & $3.58 \mathrm{ab}$ & $3.33 \mathrm{ab}$ & $8.26 \mathrm{~b}$ \\
$\mathrm{DMS}$ & 4.92 & 0.76 & 4.05 & 200.54 & 5.24 & 2.99 & nd & 0.48 & 0.88 & 0.77 & 1.81 \\
$\mathrm{CV}(\%)$ & 8.03 & 9.81 & 5.49 & 19.25 & 31.83 & 7.1 & nd & 25.77 & 17.37 & 16.72 & 15.6 \\
\hline
\end{tabular}

Medias con la misma lateral en columna son iguales estadísticamente de acuerdo con la prueba LSD $(p \leq 0.05)$. AP= altura de planta; $\mathrm{DTP}=$ diámetro de tallo principal; $\mathrm{CRC}=$ contenido relativo de clorofila en unidades $\mathrm{SPAD} ; \mathrm{AF}=$ área foliar; $\mathrm{VR}=$ volumen de raíz; $\mathrm{NBP}=$ número de botones por planta; $\mathrm{PAF}=$ porcentaje de aborto de flor; $\mathrm{MSR}=$ materia seca de raíz; $\mathrm{MST}=$ materia seca de tallo; $\mathrm{MSH}=$ materia seca de hoja; $\mathrm{MSTOT}=$ materia seca de la planta completa; DMS = diferencia mínima significativa; $\mathrm{CV}=$ coeficiente de variación; $\mathrm{nd}=$ no determinado; ${ }^{*}=$ tratamiento testigo.

La etapa de fructificación del chilhuacle tuvo una duración de 184 días con una temperatura promedio de $20.9^{\circ} \mathrm{C}$. En este periodo son importantes los componentes del rendimiento y la calidad del fruto. En cuanto al primero, las diferentes relaciones $\mathrm{NO}_{3}{ }^{-}: \mathrm{K}^{+}$evaluadas tuvieron efecto estadísticamente similar $(p \leq 0.05)$ en el número de frutos por planta $(53)$, rendimiento de materia fresca (1.416 kg planta ${ }^{-1}$ ) y seca de frutos $\left(0.253 \mathrm{~kg}_{\text {planta }}{ }^{-1}\right)$ (Cuadro 3). El número de frutos en los tallos principales varió significativamente. En este sentido, con la relación $\mathrm{NO}_{3}^{-}: \mathrm{K}^{+} 14: 5$ meq $\mathrm{L}^{-1}$ las plantas presentaron el mayor número de frutos en los tallos principales (20.4), lo que representó $54.54 \%$ más que las plantas testigo $\left(12: 1: 7 \mathrm{meq} \mathrm{L}^{-1}\right)$.

Los mismos resultados reportados en chile habanero donde la relación 14:5 meq $\mathrm{L}^{-1}$ incrementó en $15.5 \%$ el número de frutos por planta, mientras que la relación 12:1:7 meq $\mathrm{L}^{-1}$ aumentó $66.4 \%$ el rendimiento cosechando hasta $1054 \mathrm{~g} \mathrm{planta}^{-1}$ (López-Gómez et al., 2017). Las plantas de chilhuacle no mostraron diferencias por efecto de la nutrición, pero si por efecto de poblaciones en rendimiento y el número de frutos, cosechando hasta $274.14 \mathrm{~g} \mathrm{planta}^{-1}$ y 13.58 frutos en chilhuacle rojo y 434.43 g planta $^{-1}$ con 29.67 frutos planta ${ }^{-1}$ en chilhuacle negro (Urbina-Sánchez et al., 2020). 
En el caso de las plantas nutridas con 14:5 meq $\mathrm{L}^{-1}$ en comparación con la relación 14:9 meq $\mathrm{L}^{-1}$ de $\mathrm{NO}_{3}{ }^{-}: \mathrm{K}^{+}$, es interesante considerar que con la misma concentración de $\mathrm{NO}_{3}{ }^{-}\left(14 \mathrm{meq} \mathrm{L}^{-1}\right)$ pero diferente en $\mathrm{K}^{+}$(5 y 9) fue estadísticamente similar en la CRC (63.81 unidades SPAD), sólidos solubles totales $\left(9.03{ }^{\circ}\right.$ Brix $)$ y peso de materia seca de raíz $(31.1 \mathrm{~g})$. A pesar de que las plantas nutridas con la relación $\mathrm{NO}_{3}^{-}: \mathrm{K}^{+} 14: 5$ tuvieron 50\% más frutos en los tallos principales en comparación con las plantas que recibieron 14:9, éstas produjeron frutos con mayor diámetro ecuatorial (5.28\%), con tendencia a producir más peso de materia fresca $(7.59 \%)$ y seca $(8.68 \%)$. Esta relación le favorece a las plantas de chilhuacle donde la concentración de $\mathrm{NO}_{3}{ }^{-}$se mantiene constante y demandante (Larios-González et al., 2021) durante todas sus etapas, mientras que el aporte de $\mathrm{K}^{+}$aumenta la calidad y el rendimiento del cultivo (Pavón et al., 2021).

Respecto al porcentaje de aborto de flor, con ninguna de las relaciones $\mathrm{NO}_{3}{ }^{-}: \mathrm{K}^{+}$evaluadas en este experimento se logró la reducción por debajo de 50\%, ya que la variación fue del 50.41\% (10:5 meq $\mathrm{L}^{-1}$ de $\mathrm{NO}_{3}^{-}: \mathrm{K}^{+}$) hasta $70.42 \%\left(12: 9\right.$ meq $\mathrm{L}^{-1}$ de $\left.\mathrm{NO}_{3}^{-}: \mathrm{K}^{+}\right)$. Con la relación 12:7 (testigo) el porcentaje de aborto de flor fue de 65.7 mientras que con 14:9 fue de 60.85 (datos no publicados). En cuanto a la calidad de los frutos, la longitud de fruto $(60.76 \mathrm{~mm})$, grosor del exocarpo $(2.99$ $\mathrm{mm})$ y número de semillas (131) fue estadísticamente similar $(p<0.05)$ independientemente de las diferentes relaciones $\mathrm{NO}_{3}^{-}: \mathrm{K}^{+}$con que las plantas fueron tratadas.

El diámetro ecuatorial del fruto afectó significativamente por las relaciones $\mathrm{NO}_{3}{ }^{-}: \mathrm{K}^{+}$(Cuadro 3). La proporción $14: 9$ meq $\mathrm{L}^{-1}$ indujo que las plantas presentarán un diámetro con $5.64 \%$ más que el testigo (12:7), entre otras relaciones de $\mathrm{NO}_{3}{ }^{-}: \mathrm{K}^{+}(10: 7,12: 5,12: 9,14: 5,14: 7)$. En cuanto al peso de la materia fresca del mismo órgano, las plantas nutridas con 14:9 de $\mathrm{NO}_{3}^{-}: \mathrm{K}^{+}$tuvieron frutos $12.88 \%$ más pesados que los producidos con 10:7 y 12:9. La relación $\mathrm{NO}_{3}{ }^{-}: \mathrm{K}^{+} 14: 9$ también indujo que los frutos presentaran mayor materia seca en comparación con las plantas cuya solución nutritiva fue de 10 y $12 \mathrm{meq} \mathrm{L}^{-1}$ (Cuadro 3), lo cual se confirma que durante todo el ciclo productivo de chilhuacle es demandante de $\mathrm{N}\left(14 \mathrm{meq}^{-1}\right)$ y K para favorecer en la formación y en la calidad que los frutos (Taiz y Zeiger, 2010).

Los frutos de chilhuacle se comercializan en seco por lo que el tamaño y el peso de la materia seca son importantes. Al respecto, las plantas nutridas con la relación 14:9 de $\mathrm{NO}_{3}{ }^{-}: \mathrm{K}^{+}$presentaron tendencia estadística de tener mayor diámetro ecuatorial, así como de materia seca (Cuadro 3). López-Gómez et al. (2017) reportó que en chile habanero var Jaguar la relación 14:5 meq $\mathrm{L}^{-1}$ de $\mathrm{NO}_{3}{ }^{-}: \mathrm{K}^{+}$en la solución nutritiva suministrada en la etapa de fructificación incrementó el rendimiento y el peso de materia fresca del fruto. Esta diferencia en la demanda de $\mathrm{K}^{+}$, con la misma concentración de $\mathrm{NO}_{3}^{-}\left(14\right.$ meq $\left.\mathrm{L}^{-1}\right)$ puede atribuirse a la alta demanda de $\mathrm{N}$ y $\mathrm{K}$ que requieren los Capsicum, por lo que en $C$. chinense uno de los chiles más estudiados la demanda que requirió fue menor que el chilhuacle.

En este mismo sentido, Urbina-Sánchez et al. (2020) fertilizaron plantas de chilhuacle con amonio en concentraciones de $0,1.5$ y 3 meq $\mathrm{L}^{-1}$, los resultados demostraron que estadísticamente no fue significativo el peso fresco y seco de fruto, sino entre poblaciones de chilhuacle negro (18.66 y $2.13 \mathrm{~g})$ y chilhuacle rojo $(23.22$ y $2.2 \mathrm{~g})$. El contenido de sólidos solubles totales presentó diferencias significativas por efectos de las soluciones nutritivas, el tratamiento testigo (12:7 meq $\mathrm{L}^{-1}$ de $\mathrm{NO}_{3}^{-}: \mathrm{K}^{+}$) incrementó este contenido a comparación de las plantas que fueron tratadas con la relación 12:5 meq $\mathrm{L}^{-1}$ donde dicho incrementó fue de $9.46 \%$ más que los frutos de las plantas nutridas con una nutrición diferente. En cuanto al contenido de solidos solubles totales (SST), Urbina-Sánchez et al. (2020) reportó mayor contenido en chilhuacle rojo donde a $0 \mathrm{meq} \mathrm{L}^{-1}$ de 
$\mathrm{NH}_{4}{ }^{-}$produjo $10.2^{\circ}$ Brix, mientras que a 3 meq $\mathrm{L}^{-1}$ de $\mathrm{NH}_{4}$ el contenido de SST fue de $10^{\circ} \mathrm{Brix}$. En chilhuacle negro presentaron en promedio $9.5^{\circ}$ Brix con un aporte de 3 meq $\mathrm{L}^{-1}$ de $\mathrm{NH}_{4}$, mismos resultados obtenidos en este estudio.

En cuanto al crecimiento de las plantas, no se manifestaron diferencias estadísticas $(p \leq 0.05)$ en el área foliar estimada (1 $384 \mathrm{~cm}^{2}$ planta ${ }^{-1}$ ) y acumulación de materia seca en hojas (12.6 g), pero fue diferente en cuanto al contenido relativo de clorofila. Las plantas nutridas con $14: 9$ de $\mathrm{NO}_{3}{ }^{-}: \mathrm{K}^{+}$ presentaron contenido relativo de clorofila estadísticamente similar que el testigo (12:7) pero $30.21 \%$ menos en comparación con las nutridas con 10:9. Los datos anteriores indican que el aumento de la concentración de $\mathrm{N}_{-} \mathrm{NO}_{3}{ }^{-}$en la solución nutritiva no incrementa de forma directa la clorofila ya que su efecto se modificó por el nivel del $\mathrm{K}^{+}$(Cuadro 3). Con relación a lo anterior, la disponibilidad de $\mathrm{N}$ se modifica la relación con $\mathrm{K}$ como en chile habanero una concentración de 12 y 7 meq L ${ }^{-1}$ aumentó el contenido de clorofila en las hojas, mientras que disminuyó cuando las plantas fueron regadas con 10 meq $\mathrm{L}^{-1}$ de $\mathrm{NO}_{3}^{-}: \mathrm{K}^{+}$(López-Gómez et al., 2017).

Si bien la clorofila en las plantas es importante porque es el pigmento responsable de la captación de la energía luminosa durante el proceso de la fotosíntesis y su concentración tiene relación directa con la intensidad del color verde (Rincón y Ligarreto, 2010; IICA. 2019), también es fundamental la eficiencia fotosintética y la partición de fotoasimilados favoreciendo el órgano de interés. Respecto a la producción de materia seca, en hojas, tallos y en la planta completa fue estadísticamente similar ( $p \leq 0.05$ ) entre tratamientos con valores de $12.6 \mathrm{~g}, 124.4 \mathrm{~g}$ y $164.9 \mathrm{~g}$, respectivamente en raíz, con la relación $\mathrm{NO}_{3}{ }^{-}: \mathrm{K}^{+} 10: 7$ las plantas acumularon $74.78 \%$ en comparación con la raíz de las plantas nutridas con la relación 12:7 (Cuadro 3). Se considera que el efecto observado en las plantas de los diferentes tratamientos se debió a la relación $\mathrm{NO}_{3}^{-}: \mathrm{K}^{+}$de la solución nutritiva puesto que todas las plantas estuvieron aleatorizadas en el mismo invernadero.

Cuadro 3. Efecto de las relaciones $\mathrm{NO}_{3}^{-}: \mathrm{K}^{+}$de la solución suministrada en la etapa de fructificación del chilhuacle sobre el contenido relativo de clorofila, peso de materia seca de raíz, número de frutos y calidad del fruto.

\begin{tabular}{cccccccc}
\hline $\begin{array}{c}\text { Relaciones } \\
\mathrm{NO}_{3}^{-}: \mathrm{K}^{+}\left(\mathrm{meq} \mathrm{L}^{-1}\right)\end{array}$ & CRC & $\begin{array}{c}\text { PMSR } \\
(\mathrm{g})\end{array}$ & NFTP & $\begin{array}{c}\text { DEF } \\
(\mathrm{mm})\end{array}$ & $\begin{array}{c}\text { PMFF } \\
(\mathrm{g})\end{array}$ & $\begin{array}{c}\text { PMSF } \\
(\mathrm{g})\end{array}$ & $\begin{array}{c}\text { SST } \\
\left({ }^{\circ} \mathrm{Brix}\right)\end{array}$ \\
\hline $10: 5$ & $70.78 \mathrm{~cd}$ & $39.8 \mathrm{ab}$ & $18.8 \mathrm{ab}$ & $53.59 \mathrm{ab}$ & $39.82 \mathrm{ab}$ & $6.87 \mathrm{ab}$ & $8.73 \mathrm{ab}$ \\
$10: 7$ & $75.48 \mathrm{abc}$ & $41.6 \mathrm{a}$ & $14.8 \mathrm{bcd}$ & $52.01 \mathrm{~b}$ & $37.09 \mathrm{~b}$ & $6.75 \mathrm{ab}$ & $8.78 \mathrm{ab}$ \\
$10: 9$ & $89.36 \mathrm{a}$ & $31 \mathrm{ab}$ & $14.8 \mathrm{bcd}$ & $53.14 \mathrm{ab}$ & $39.47 \mathrm{ab}$ & $6.82 \mathrm{ab}$ & $8.99 \mathrm{ab}$ \\
$12: 5$ & $83.5 \mathrm{ab}$ & $33.4 \mathrm{ab}$ & $18.4 \mathrm{abc}$ & $51.77 \mathrm{~b}$ & $38.92 \mathrm{ab}$ & $6.69 \mathrm{ab}$ & $8.56 \mathrm{~b}$ \\
$12: 7^{*}$ & $59.32 \mathrm{~d}$ & $23.8 \mathrm{~b}$ & $13.2 \mathrm{~cd}$ & $51.87 \mathrm{~b}$ & $39.08 \mathrm{ab}$ & $7.03 \mathrm{ab}$ & $9.37 \mathrm{a}$ \\
$12: 9$ & $58.8 \mathrm{~d}$ & $26.4 \mathrm{ab}$ & $11.6 \mathrm{~d}$ & $51.01 \mathrm{~b}$ & $36.74 \mathrm{~b}$ & $6.51 \mathrm{~b}$ & $8.86 \mathrm{ab}$ \\
$14: 5$ & $65.26 \mathrm{~cd}$ & $35 \mathrm{ab}$ & $20.4 \mathrm{a}$ & $52.05 \mathrm{~b}$ & $38.71 \mathrm{ab}$ & $6.68 \mathrm{ab}$ & $9.21 \mathrm{ab}$ \\
$14: 7$ & $62.36 \mathrm{~cd}$ & $29 \mathrm{ab}$ & $16.4 \mathrm{abcd}$ & $51.86 \mathrm{~b}$ & $38 \mathrm{ab}$ & $6.64 \mathrm{ab}$ & $9.26 \mathrm{ab}$ \\
$14: 9$ & $62.36 \mathrm{~cd}$ & $27.2 \mathrm{ab}$ & $13.6 \mathrm{bcd}$ & $54.8 \mathrm{a}$ & $41.65 \mathrm{a}$ & $7.26 \mathrm{a}$ & $8.85 \mathrm{ab}$ \\
$\mathrm{DMS}$ & 14.79 & 16.82 & 5.28 & 2.63 & 4.05 & 0.73 & 0.76 \\
$\mathrm{CV}(\%)$ & 16.47 & 40.92 & 9.76 & 3.90 & 8.11 & 8.3 & 6.58 \\
\hline
\end{tabular}

Medias con la misma lateral en columna son iguales estadísticamente con la prueba LSD $(p \leq 0.05)$. CRC $=$ contenido relativo de clorofila en unidades SPAD; PMSR= peso de materia seca de raíz; NFTP0= número de frutos en tallos principales; $\mathrm{DEF}=$ diámetro ecuatorial de fruto; $\mathrm{PMFF}=$ peso de materia fresca de fruto; $\mathrm{PMSF}=$ peso de materia seca de fruto; $\mathrm{SST}=$ sólidos solubles totales; $\mathrm{DMS}=$ diferencia mínima significativa; $\mathrm{CV}=$ coeficiente de variación; ${ }^{*}=$ testigo. 


\section{Conclusiones}

En la etapa vegetativa, la concentración con 14 meq $\mathrm{L}^{-1}$ de $\mathrm{NO}_{3}{ }^{-}$se favoreció la altura, el diámetro de tallo, volumen de raíz, la materia seca del tallo y de la planta completa. En la etapa reproductiva, las relaciones $\mathrm{NO}_{3}{ }^{-}: \mathrm{H}_{2} \mathrm{PO}_{4}{ }^{-}: \mathrm{SO}_{4}{ }^{2-}$ de la solución nutritiva modificó significativamente el número de botones por planta. Con 10:0.75:9.25 y 12:1:7 meq $\mathrm{L}^{-1}$ (testigo) se obtuvo la mayor cantidad; sin embargo, 10:0.75:9.25 meq $\mathrm{L}^{-1}$ provocó el menor porcentaje de aborto de flor. En la etapa de fructificación, la relación 14:9 meq $\mathrm{L}^{-1}$ de $\mathrm{NO}_{3}^{-}: \mathrm{K}^{+}$influyó de manera diferente en la calidad de los frutos de chilhuacle en presentar mayor diámetro, al igual que el peso de la materia fresca y seca.

\section{Literatura citada}

Azofeifa, Á. y Moreira, M. A. 2004. Análisis de crecimiento del chile jalapeño (Capsicum annuum L. $c v$ Hot), en Alajuela, Costa Rica. Agron. Costarr. 28(1):57-67. http://www.redalyc.org/ pdf/436/43628106.pdf.

Corrales-Maldonado, C. G.; Vargas-Arispuro, I.; Vallejo-Cohén, S. y Martínez-Téllez, M. A. 2014. Deficiencia de azufre en suelos cultivables y su efecto en la productividad. Biotecnia. 16(1):38-44. http://www.repositorioinstitucional.uson.mx/handle/unison/4265.

Fajardo-Rebollar, E.; Estrada, K.; Grande, R.; Ek Ramos, M. J.; Ruiz, V. G.; Villegas-Torres, O. G. and Camino, C. D. 2021. Bacterial and fungal microbiome profiling in chilhuacle negro chili (Capsicum annuum L.) associated with fruit rot disease. Plant Dis. https://doi.org/10.1094/PDIS-09-20-2098-RE.

García-Gáytan, V.; Gómez, M. F. C.; Trejo, T. L. I.; Baca, C. G. A. and Garcia, M. S. 2017. The chilhuacle chili (Capsicum annuиm L.) in Mexico: description of the variety, its cultivation, and uses. Inter. J. Agron. 2017. https://www.hindawi.com/journals/ija/2017/5641680/.

IICA. 2019. Instituto Interamericano de Cooperación para la Agricultura. Guía para la identificación y análisis de las posibilidades de negocios de la Bioeconomía. Costa Rica. 200 p.

Larios-González, R. C.; García, C. L.; Ríos, M. J.; Avalos, E. C. D. S. y Castro, S. J. R. 2021. Pérdidas de nitrógeno por volatilización a partir de dos fuentes nitrogenadas y dos métodos de aplicación. Siembra. 8(2): e2575. https://doi.org/10.29166/siembra.v8i2.2475.

López, L. P. y Pérez, D. B. 2015. El chile Huacle (Capsicum annuum sp.) en el estado de Oaxaca, México. Rev. Agroproductividad. 8(1):35-39. http://www.revista-agroproductividad.org/ index.php/agroproductividad/article/view/636.

López, L. P.; Rodríguez, H. R. y Bravo, M. E. 2016. Impacto económico del chile Huacle (Capsicum annuum L.) en el estado de Oaxaca. Rev. Mex. Agron. 20(38):317-328. Doi:10.22004 / ag.econ.239292.

López-Gómez, J. D.; Villegas, T. O. G.; Nava, H. S.; Rodríguez, M. A.; López, P. J. y Fernández, E. M. 2017. Rendimiento y calidad del chile habanero (Capsicum chinense Jacq.) por efecto del régimen nutrimental. Rev. Mex. Cienc. Agríc. 8(8):1747-1758. http://www.redalyc.org/ articulo.oa?id=263153822009.

Luna-Fletes, J. A.; Cruz-Crespo, E. y Can-Chulim, Á. 2021. Piedra pómez, tezontle y soluciones nutritivas en el cultivo de tomate Cherry. Terra Latinoam. 39:1-12. https://doi.org/ 10.28940/terra.v39i0.781.

Moreno-Pérez, E. C.; Sánchez-Del Castillo, F.; Gaspar, F. J. M.; Ramírez-Árias, A. y BerylColinas-León, M. T. 2019. Rendimiento de pimiento morrón (Capsicum annuum L.) por poda floral selectiva y despunte de yemas laterales en la cuarta bifurcación. Agrociencia. 53(5):697-707. https://agrociencia-colpos.mx/index.php/agrociencia/article/view/1837. 
Nazar, R.; Iqbal, N.; Masood, A.; Syeed, S. and Khan, N. A. 2011. Understanding the significance of sulfur in improving salinity tolerance in plants. Environ. Exp. Bot. 70(2-3):80-87. https://doi.org/10.1016/j.envexpbot.2010.09.011.

Noh, M. J.; Borges, G. L.; y Soria, F. M. 2010. Composición nutrimental de biomasa y tejidos conductores en chile habanero (Capsicum chinense Jacq.). Trop. Subtrop. Agroecosys. 12(2):219-228. https://www.redalyc.org/articulo.oa?id=93913070003.

Pavón, T. L. D.; Fernández, B. J. A. y Graupera, G. X. J. 2021. Efectos de fertilización potásica en el cultivo de pimiento (Capsicum annum L.) var híbrido Nathalie. Alfa Rev. de Investigación en Ciencias Agronómicas y Veterinaria. 5(13):78-90. https://doi.org/ 10.33996/revistaalfa.v5i13.99.

Peil, R. M. y Gálvez, J. L. 2005. Reparto de materia seca como factor determinante de la producción de las hortalizas de fruto cultivadas en invernadero. Agrociencia 11(1):05-11. http://dx.doi.org/10.18539/CAST.V11I1.1171; https://periodicos.ufpel.edu.br/ojs2/index. php/CAST/article/view/1171/966.

Rincón, C. Á. y Ligarreto, G. A. 2010. Relación entre nitrógeno foliar y el contenido de clorofila, en maíz asociado con pastos en el Piedemonte Llanero colombiano. Corpoica. Ciencia y Tecnología Agropecuaria. 11(2):122-128. http://www.redalyc.org/pdf/4499/449945029 003.pdf.

Sieiro, Miranda, G. L.; González Marrero, A. N.; Rodríguez Lema, E. L.; Rodríguez Regal, M. 2020. Efecto de los macroelementos primarios en la susceptibilidad a enfermedades. Centro Agrícola. 47(3):66-74. http://scielo.sld.cu/scielo.php?pid=S0253-57852020000300066\& script $=$ sci_abstract \&tlng $=$ es.

Steiner, A. A. 1984. The universal solution. ISOSC. In: Proceedings of $6^{\text {th }}$ International Congress on Soilles Culture. Lunteren, The Netherlands. 633-649 pp.

Taiz, L. and Zeiger, E. 2010. Plant physiology $5^{\text {th }}$ (Ed.). Sunderland. Sinauer Assoc, Inc., Publishers, Sunderland Masschusetts. 764 p.

Urbina-Sánchez, E.; Cuevas-Jiménez, A.; Reyes-Alemán, J. C.; Alejo-Santiago, G.; ValdezAguilar, L. A.; Vázquez-García, L. M. 2020. Solución nutritiva adicionada con $\mathrm{NH}_{4}{ }^{+}$para producción hidropónica de chile huacle (Capsicum annuum L.). Rev. Fitotec. Mex. 43(3):291-298. https://doi.org/10.35196/rfm.2020.3.291. 